\title{
Analysis of Long-Chain Fatty Acyl \\ Coenzyme A Thioesters by Negative \\ Ion Fast-Atom Bombardment Mass \\ Spectrometry and Tandem \\ Mass Spectrometry
}

\author{
Joseph A. Zirrolli, Pat Wheelan, and Robert C. Murphy \\ National Jewish Center for Immunology and Respiratory Medicine, Denver, Colorado, USA
}

\begin{abstract}
Long-chain acyl Coenzyme A (CoA) is essentially composed of three major chemical groups, fatty acyl-, phosphopantetheino-, and 3',5',-adenosine diphospho-moieties. The negative ion fast-atom bombardment mass spectrometry spectra of long-chain acyl CoA thioesters were characterized by the formation of abundant $[\mathrm{M}-\mathrm{H}]^{-}$and two distinct classes of fragment ions, one class which retained the acyl group and another class which is related to CoA that contains the phosphopantethene and adenine. The ions which retained the acyl group in the spectrum of palmitoyl CoA appeared at $m / z$ 675, 657, 595, and 577 and were found to decompose by loss of alkylketene observed at $m / z 357$ and 339 . Those ions which retained the adenine group were observed at $m / z 426$ and 408 . In contrast to these ions observed following fast-atom bombardment ionization, tandem mass spectrometry of the $[\mathrm{M}-\mathrm{H}]^{-}$, from palmitoyl $\operatorname{CoA}(m / z$ 1004), yielded the adenine-containing ions as major products and the acyl-containing ions were of low abundance or not detected. These results suggested that the formation of many characteristic ions observed in direct $F A B$ analysis occurred during the desorption process. The unique relationship between ions which involved the transition from acyl-containing ions to only CoA-containing ions by the loss of alkylketene allowed the development of tandem mass spectrometry protocols for the analysis of acyl CoA mixtures. Precursor scans of either $m / z 357$ or 339 yielded the identification of each species in a complex mixture. Identification of specific species was obtained with a neutral loss scan of the mass for a specific alkylketene. (J Am Soc Mass Spectrom 1994, 5, 416-424)
\end{abstract}

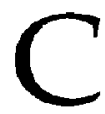
oenzyme $A(C O A)$ thioesters of long-chain fatty acids (acyl $\mathrm{CoA}$ ) are essential intermediates in all aspects of fatty acid synthesis [1], degradation [2], and phospholipid biosynthesis [3]. Acyl CoA thioesters are thought to play important roles in many cellular regulatory processes including biosynthesis of the lipid mediator platelet activating factor [4], and intermediates of leukotriene $[5,6]$ and prostaglandin metabolism [7]. In addition, acyl CoA thioesters are also formed with xenobiotics in the pathway of conjugation to endogenous amino acids [8]. Because of the atomic size of the sulfur atom in the thioester compared to the oxygen atom in esters, there is less $\pi$-electron overlap and a destabilization of the thioester bond relative to that of an ester. This makes the thiolate anion a better leaving group in nucleophilic displacement reactions that are catalyzed by various enzymes that use the acyl CoA derivative.

Address reprint requests to Dr. Robert C. Murphy, Department of Pediatrics, National Jewish Center, 1400 Jackson Street, Denver, CO 80206 .
Long-chain acyl COA is essentially composed of three major chemical groups: fatty acyl-, phosphopantetheino-, and $3^{\prime}, 5^{\prime}$-adenosine diphospho-moieties (ADP). Several mass spectrometric strategies have been used for the analysis of acyl CoAs. Long-chain fatty alcohols formed from the reduction of acyl COA esters have been analyzed by electron capture negative ion gas chromatography mass spectrometry as the pentafluorobenzoyl esters [9]. While this method is reported to be quite sensitive, it has the disadvantages inherent with chemical workup and derivatization. Analysis of intact acyl CoAs has involved direct and continuous-flow fast-atom bombardment mass spectrometry (FAB/MS) [10-12]. These fast-atom bombardment (FAB) studies were based upon positive ionization modes. Both $[\mathrm{M}+\mathrm{H}]^{+}$and fragment ions indicative of the $\mathrm{COA}$ and acyl group were observed, and the base peak of the spectrum was protonated adenine, $m / z$ 136. A recent report using tandem mass spectrometry of positive ions from 3-keto-2-propylpentanoyl-CoA indicated the utility of this approach to probe detailed structural features in this 
unique CoA ester [13]. Little work has been performed to evaluate the efficacy of negative ionization of intact acyl CoAs. One previous study did report that abundant $[\mathrm{M}-\mathrm{H}]^{-}$ions were produced from $\mathrm{LTB}_{4}-\mathrm{CoA}$ [6]; however, this study did not describe any other ion formation. For these reasons we have investigated the negative ion FAB/MS and fast-atom bombardment tandem mass spectrometry (FAB/MS/MS) behavior of long-chain acyl CoAs.

\section{Methods}

Standard long-chain acyl CoA species, in their free acid form, were obtained from Sigma Chemical Company (St. Louis, MO). Individual and mixture of standards were dissolved in methylene chloride/methanol $(2 / 1)$ to give a final concentration of $1 \mathrm{ug} / \mathrm{ul}$ per each compound. Aliquots $(1 \mathrm{ul})$ were applied to the FAB probe using a variety of matrices (reagent grade) as described in the Results. $\left[{ }^{2} \mathrm{H}\right]_{10}-(\mathrm{N}, \mathrm{O})$-Palmitoyl-CoA was prepared by dissolving $10 \mathrm{ug}$ of palmitoyl-CoA in 100 ul of ethanol-OD (99.5\% D, Aldrich, Milwaukee, WI), evaporation of the solvent in vacuo, and repeating the procedure three times. $\left[{ }^{2} \mathrm{H}\right]_{10}-(\mathrm{N}, \mathrm{O})$-Palmitoyl-CoA was analyzed using $\left[{ }^{2} \mathrm{H}\right]_{3}$-glycerol $(98 \% \mathrm{D}$, Aldrich, Milwaukee, WI) as the FAB matrix. The most abundant isotopic species observed was $\left[{ }^{2} \mathrm{H}\right]_{10}-(\mathrm{N}, \mathrm{O})-$ palmitoyl-CoA which was selected for collision-induced dissociation (CID) and analysis by tandem mass spectrometry. Other species were observed at lower abundances containing eight and nine deuterium atoms indicating incomplete deuterium exchange, but these species did not interfere with the CID experiments.

Analyses were performed on a Finnigan TSQ 70 mass spectrometer (Finnigan Corporation, San Jose, CA) equipped with a FAB gun from Ion Tech (Teddington, England). Scans were obtained over the range $m / z 200$ to $1200 \mathrm{u}$ at the rate of $2 \mathrm{~s} / \mathrm{scan}$. The FAB gun was operated with xenon as the particle source at 6 $\mathrm{keV}$. Tandem mass spectrometry employed a collision energy of $30 \mathrm{eV}$ (laboratory frame of reference) with argon as the collision gas at a pressure of 0.5 mTorr.

\section{Results}

\section{$F A B / M S$ (Source-formed ions)}

With negative ion FAB/MS desorption and ionization each group, fatty acyl-, phosphopantetheino-, and $3^{\prime}, 5^{\prime}$-adenosine diphospho-moieties (Scheme I) exerted an influence to yield fragment ions characteristic of that group. Indeed, long-chain acyl $\mathrm{CoA}$ thioesters produced abundant $[\mathrm{M}-\mathrm{H}]^{-}$ions and two distinct classes of fragment ions, one class retaining the acyl group (designated $I-V$ ) and the other related to coenzyme $A$ and for adenosine diphosphate (designated $A-E)$. The class of ions $(A-E)$ related to the COA or ADP groups are common to all acyl CoAs while acylcontaining ions $(I-V)$ shift in mass according to the mass of the acyl group. This is illustrated in Figure 1

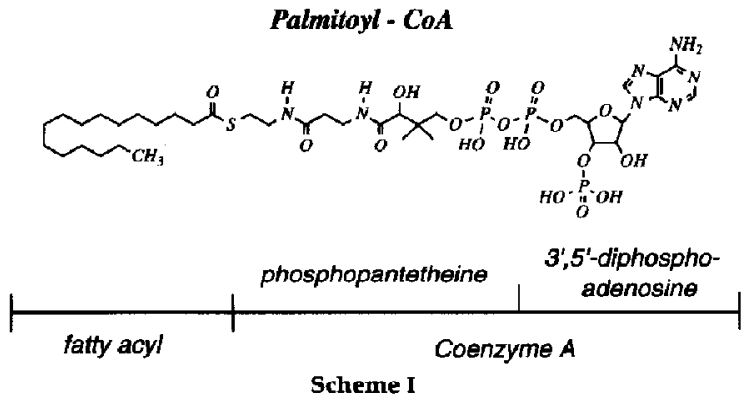

with the negative ion spectra of palmitoyl- and arachidonyl-CoA which displayed $[\mathrm{M}-\mathrm{H}]^{-}$at $m / z 1004$ and 1052, respectively. (Even though the number of hydrogen atoms in acyl $\mathrm{COA}$ species is relatively high (66-74 protons), the exact masses observed for molecular ion species is only $0.2-0.4$ above the nominal mass because of the number of mass deficient heteroatoms also present. The exact molecular masses for palmitoyl and arachidonoyl CoA are 1005.3 and 1052.3, respectively. In this publication we will report the nominal masses for observed ions rather than the exact masses.) The major fragment ions of each class are described below. The assignment of ion structures is supported by appropriate mass shifts between the spectra of palmitoyl- and $\left[{ }^{2} \mathrm{H}\right]_{10}-(\mathrm{N}, \mathrm{O})$-palmitoyl-CoA as depicted below in specific ion structures.
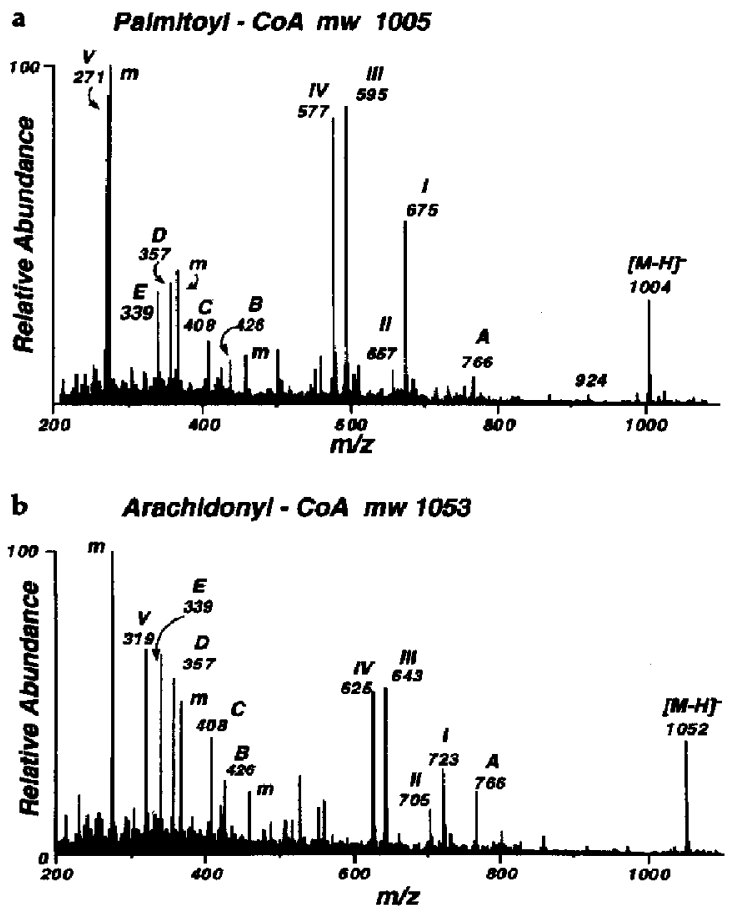

Figure 1. Negative ion FAB/MS of (a) palmitoyl COA and (b) arachidonyl CoA obtained in glycerol matrix; letter $m$ designates matrix ions. 
Formation of most negative ions can be rationalized by an initial hydrogen abstraction from and charge localization on one of the phosphate hydroxyl groups. Fragmentation of the 5'-phosphate ester (adenosine3'-monophosphate (AMP)) bond of palmitoyl-CoA with charge retention on the acyl diphosphopantetheine group yields the ion observed at $m / z 675$ (I), while $m / z 657$ (II) nominally represents an additional loss of $\mathrm{H}_{2} \mathrm{O}$. In the spectrum of $\left[{ }^{2} \mathrm{H}\right]_{10}$-(N,O)-palmitoyl-CoA these ions were shifted to $m / z 680$ and 660, respectively, corresponding to a loss of $\mathrm{D}, \mathrm{O}$. This loss of water involving exclusively exchangeable protons is consistent with the formation of a cyclic phosphate ester bond.

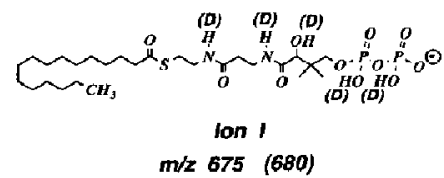

$$
\underbrace{(1 / 2)}_{\text {IOn } \|}
$$

The ions observed at $m / z 595$ and 426 ( 111 and $B$, respectively) may be formed by fragmentation on either side of the central oxygen in the diphospho ester bridge between AMP and acyl pantetheine. Thus $I I$, an acyl diagnostic ion, may be formed with charge retention on the acylphosphopantetheine and $B, a \operatorname{CoA}$ diagnostic ion, may be formed with charge retention on the 5'-phospho-AMP.
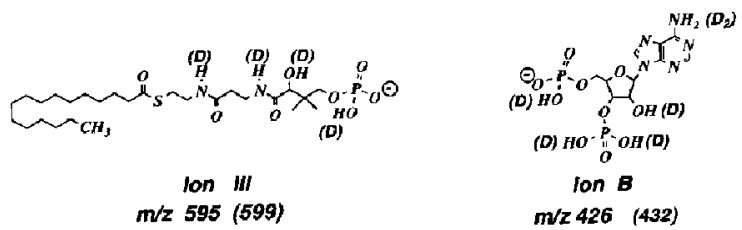

lons $C(m / z 408)$ and $I V(m / z 577)$ are most likely dehydrated analogs of $B$ and $I I I$, respectively. In the spectrum of $\left[{ }^{2} \mathrm{H}\right]_{10}-(\mathrm{N}, \mathrm{O})$-palmitoyl-CoA these ions were shifted to $m / 2599$ (III), 579 (IV), 432 (B), and 412 (C), again consistent with the formation of cyclic phosphate esters in $I V$ and $C$.
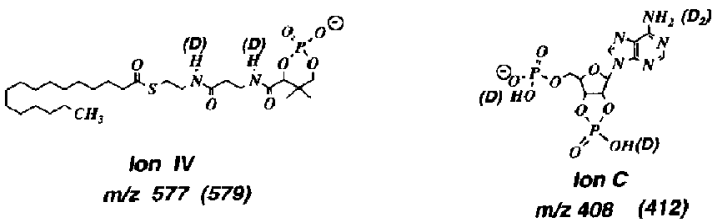

The ions observed at $m / z 357(D)$ and $m / z 339$ (E) in the mass spectrum of palmitoyl-CoA were also observed in the mass spectra of all other acyl CoAs studied (Figure 1 and Table 1). One reasonable pathway for the formation of these ions $(D)$ and $(E)$ is by loss of alkylketene from the acyl phosphopantetheine

\begin{tabular}{|c|c|c|c|c|c|}
\hline \multirow[b]{2}{*}{ Fatty Acyl Group ${ }^{b}$} & \multicolumn{5}{|c|}{$\begin{array}{l}\text { Abundance of CoA Diagnostic Fragment lons from Acyl CoA } \\
\text { Fragment lon }\end{array}$} \\
\hline & $A$ & $\boldsymbol{B}$ & $C$ & $D$ & $E$ \\
\hline & $m / 2766$ & $\mathrm{~m} / \mathrm{z} 426$ & $m / 7408$ & $\mathrm{~m} / \mathrm{z} 357$ & $\mathrm{~m} / \mathrm{z} 339$ \\
\hline $16: 0$ & 12 & 14 & 19 & 53 & 47 \\
\hline $17: 0$ & 7 & 16 & 23 & 53 & 65 \\
\hline $18: 1$ & 15 & 30 & 58 & 33 & 93 \\
\hline 18:0 & 16 & 12 & 24 & 38 & 60 \\
\hline 19:0 & 12 & 18 & 25 & 50 & 49 \\
\hline $20: 4$ & 32 & 37 & 63 & 88 & 93 \\
\hline $20: 0$ & 28 & 9 & 17 & 43 & 70 \\
\hline
\end{tabular}
ions, III and IV. The significance of this transition is

Table 1. Abundance of source-formed fragment ions from fatty acyl CoAs

\begin{tabular}{|c|c|c|c|c|c|c|}
\hline & \multirow[b]{2}{*}[\mathbf{M}-\mathbf{H}]{$^{-}$} & \multicolumn{4}{|c|}{ Fragment lon } & \multirow[b]{2}{*}{$V$} \\
\hline & & I & II & III & IV & \\
\hline $16: 0$ & $1004(42)$ & $675(33)$ & $6 b 7(12)$ & $59 b(116)$ & $577(106)$ & $271(100)$ \\
\hline $17: 0$ & $1018(23)$ & $689(32)$ & $671(12)$ & $609(68)$ & $591(79)$ & $285(100)$ \\
\hline $18: 1$ & $1030(40)$ & $701(28)$ & $683(16)$ & $621(48)$ & $603(149)$ & $297(100)$ \\
\hline $18: 0$ & $1032(40)$ & $703(38)$ & $685(14)$ & $623(52)$ & $605(90)$ & $299(100)$ \\
\hline 190 & $1046(28)$ & $717(25)$ & $699(10)$ & $637(58)$ & $619(47)$ & $313(100)$ \\
\hline $20: 4$ & $1052(54)$ & $723(46)$ & $705(24)$ & $643\langle 95\rangle$ & $625(73)$ & $319(100)$ \\
\hline 200 & $1060(20)$ & 731 (31) & $713(14)$ & $651(41)$ & $633(55)$ & $327(100)$ \\
\hline
\end{tabular}

Abundance of Fatty Acyl Diagnostıc Fragment lons from Acyl CoA

aspectra were obtained in glycerol and abundances listed are normalized to ion $v(100)$.

Acyl nomenclature cc:b, where cc is the carbon chain length and $b$ is the total number of double bonds

cData are listed as mess-to-charge ratio values with relative abundances in parentheses. 
important because the fatty acyl containing ions (III and $I V$ ) decompose to CoA diagnostic ions ( $D$ and $E$, respectively) which lack the fatty acyl group.

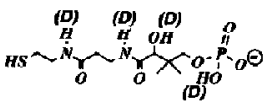

$$
\begin{aligned}
& \text { ion } D \\
& m / z 357 \text { (361) }
\end{aligned}
$$

$$
\underbrace{\text { (D) } E}_{m / 2339}
$$

The other two ions, $A$ and $V$, observed at $m / z 766$ and 271 in the spectrum of palmitoyl-CoA (Figure 1) correspond to fragmentation on either side of the thioester bond. Loss of alkylketene from $\left[\mathrm{M}-\mathrm{H}^{-}\right.$ion yields $\mathrm{A}, m / z 766$, an ion analogous to the $[\mathrm{M}-\mathrm{H}]^{-}$ from free CoA. Interestingly, this ion shifted by $9 \mathrm{u}$ to $m / z 775$ in the spectrum of the deuterated analog indicating the loss of one deuteron. Scission of the sulfur bond between thioester and pantetheine yields another important acyl diagnostic ion $V$, the acyl thiolate anion.

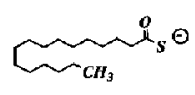

\section{Ion $V$} $m / 2271(271)$

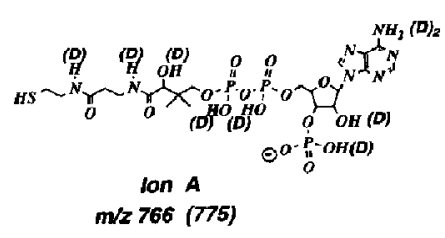

A series of seven saturated and unsaturated long chain-acyl CoAs (16:0, 17:0, 18:0, 18:1, 19:0, 20:0, and 20:4) were studied under negative ion $F A B / M S$ using a variety of matrices. The negative ion FAB mass spectra of this series of long-chain acyl CoAs are summarized in Table 1 (glycerol matrix). The most abundant fragment ions are the acyl-specific ions $I I, I V$, and $V$, and the $C O A$ diagnostic ions $D$ and $E$. The least abundant fragment ions formed were the $C O A$ related $A, B$, and $C$ ions.

The matrices employed included thioglycerol, glycerol, diethanolamine, and triethanolamine. In general, spectra containing abundant $[\mathrm{M}-\mathrm{H}]^{-}$and fragment ions, in particular abundant type $V$ ions, were obtained with the more basic matrices, diethanolamine and triethanolamine. Spectra generated in the presence of the relatively more acidic matrix, thioglycerol, also yielded abundant $\left[\mathrm{M}-\mathrm{H}^{-}\right.$ions and more abundant acyl-containing ions $I I I$ and $I V$, but less abundant thiolate type anions $V$ (Table 2).
From the perspective of identifying specific acylcontaining CoA thioesters, negative ion FAB/MS demonstrated good performance and prompted further study of their CID behavior to evaluate specific decompositions for the analysis of complex mixtures.

\section{$F A B / M S / M S$ :}

Tandem mass spectrometry included product ion, precursor ion, and neutral loss scans following CID studies of the long-chain acyl CoAs to investigate the formation of the fragment ions observed in FAB/MS spectra. Product ions formed from CID of the major source-formed ions from palmitoyl CoA are summarized in Table 3 and listed in comparison with the FAB/MS spectrum. Product ion spectra from the other acyl CoA species were qualitatively similar.

$[M-H]^{-}(m / z$ 1004). As shown in Figure 2 , the most abundant product ions formed from CID of the $[\mathrm{M}-\mathrm{H}]^{-}$of palmitoyl-CoA are the CoA class ions, $B$ and $C$ ( $m / z 426$ and 408 , respectively). The acyl containing ions were either of low abundance, $I(\mathrm{~m} / \mathrm{z}$ 675), II $(m / z 657)$, and $I V(m / z 577)$ or completely absent, III $(m / z 595)$ and $V(m / z 271)$. Also absent were the CoA ions $D$ and $E(m / z \quad 357$ and 339 , respectively), which are derived from acyl-containing ions by the loss of alkylketene. The product ion spectrum is in contrast to the FAB/MS analysis in which the most abundant ions observed were acyl-containing fragments and the least abundant ions were CoA related (Figure 1a). Other minor product ions were observed at $m / z$ 924, loss of $80 \mathrm{u}\left(\mathrm{HPO}_{3}\right)$ from $[\mathrm{M}-\mathrm{H}]^{-}$, and two fragments not seen in the $\mathrm{FAB}$ /MS spectrum, $m / z 328$ and 273. These two ions, $m / z 328$ and 273, most likely derive from ions $B(\mathrm{~m} / z 426)$ and $C(\mathrm{~m} / z$ 408) by losses of $98 \mathrm{u}\left(\mathrm{H}_{2} \mathrm{PO}_{4}\right)$ and $135 \mathrm{u}$ (adenine), respectively.

Ion $I(m / z 675)$. The product ion spectrum of $I$ from palmitoyl CoA is shown in Figure 3. Two abundant ions $m / z 577$ and 339 ( $I V$ and $E$, respectively) are formed by an initial loss of $\mathrm{H}_{3} \mathrm{PO}_{4}(98 \mathrm{u})$ and the loss of $\mathrm{H}_{3} \mathrm{PO}_{4}$ followed by the loss of hexadecylketene (238 1), respectively. These losses are supported by the CID

\begin{tabular}{|c|c|c|c|c|c|c|c|c|c|c|c|}
\hline Matrix & {$[\mathrm{M}-\mathrm{H}]^{-}$} & $A$ & $B$ & $C$ & $D$ & $E$ & 1 & II & III & $I V$ & $\bar{v}$ \\
\hline$m / z$ & 1004 & 766 & 426 & 408 & 357 & 339 & 675 & 657 & 595 & 577 & 271 \\
\hline TEA & 27 & 6 & 14 & 14 & 12 & 21 & 12 & 7 & 34 & 37 & 100 \\
\hline DEA & 26 & 7 & 8 & 10 & 17 & 12 & 11 & 5 & 28 & 31 & 100 \\
\hline Glycerol & 36 & 10 & 12 & 16 & 46 & 40 & 28 & 10 & 100 & 91 & 86 \\
\hline Thioglycerol & 25 & 6 & 20 & 20 & 47 & 48 & 16 & 76 & 77 & 100 & 46 \\
\hline
\end{tabular}
of this ion $(\mathrm{m} / \mathrm{z} 680)$ from $\left[{ }^{2} \mathrm{H}\right]_{10}-(\mathrm{N}, \mathrm{O})$-palmitoyl-CoA in which both product ions lost three deuterium atoms (as ${ }^{2} \mathrm{H}_{3} \mathrm{PO}_{4}$ ) and the observed product ions, retaining

Table 2. Matrix effects on observed abundances of source-formed fragment ions from palmitoyl $C_{0} A^{a}$

\footnotetext{
aon abundances are from an averaged spectrum of five scans, nomalized to the most abundant ion.
} 


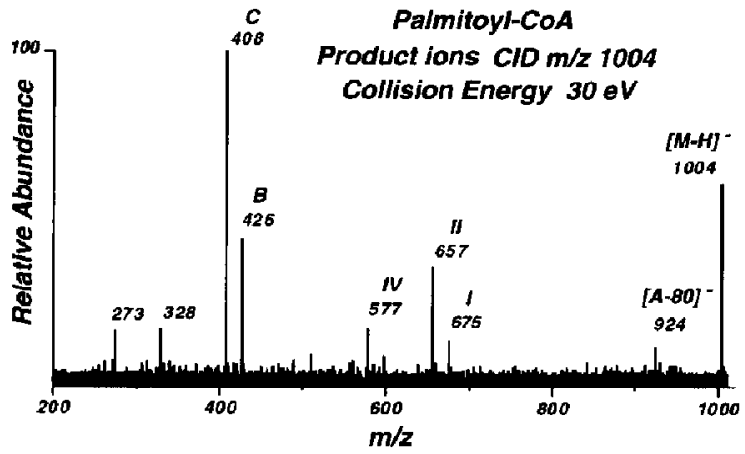

Figure 2. Product ions obtained following collision induced dissociation ( $30 \mathrm{eV}$ collision energy, $0.5 \mathrm{~m}$ 'Iorr argon) of [M $\mathrm{H}^{-}$ions of palmitoyl CoA.

two deuterium atoms, were shifted by $2 \mathrm{u}$ to $\mathrm{m} / \mathrm{z} 579$ and 341, respectively (Table 3). Also the formation of ion $E$ by loss of alkylketene was supported by the observation of this ion in the FAB/MS spectra of all other acyl CoAs studied (Table 1) as well as in their CID spectra of the respective $I$ type ions (data not shown). Other products ions, II, III, $D$, and $V(\mathrm{~m} / \mathrm{z}$ $657,595,357$, and 271 , respectively) were also observed in the FAB/MS spectrum. Of these ions, $I I I$ and $D$ were minor ions in contrast to the $\mathrm{FAB} / \mathrm{MS}$ analysis in which they were very abundant. Product ions not observed in the FAB/MS spectrum included $m / z 305$,

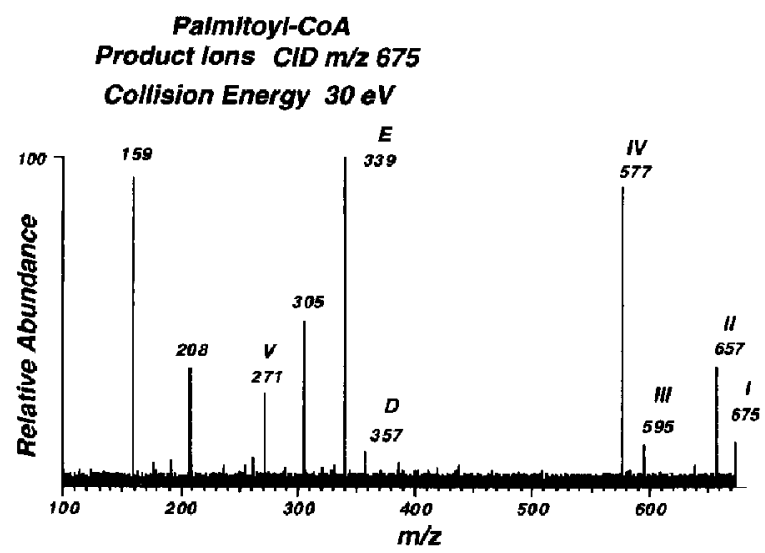

Figure 3. Product ions obtained following collision induced dissociation ( $30 \mathrm{eV}$ collision energy, 0.5 mTorr argon) of $\mathrm{m} / \mathrm{z}$ 675, Type $I$ ions from palmitoyl CoA.

208, and 159. These product ions were unchanged in observed mass in the CID of $I$ from the other longchain acyl CoAs studied and therefore did not retain the acyl group. The ion at $m / z 305$ was also observed in the CID spectra of $I I(m / z$ 657) and $I V(m / z 577)$ as listed in Table 3, and the formation of this product ion most likely involves loss of the thioacid (RCOSH, $272 \mathrm{u})$ from $I V(m / z 577)$. That this ion is shifted by only $1 \mathrm{u}$ in the CID spectrum of the deuterated analog

Table 3. FAB / MS and FAB/MS/MS ions from palmitoyl CoA

\begin{tabular}{|c|c|c|c|c|c|}
\hline \multirow[b]{2}{*}{ FAB $/ \mathrm{MS}^{\mathrm{a}, \mathrm{c}}$} & \multicolumn{5}{|c|}{ Collision Induced Dissociation lon ${ }^{a, b}$} \\
\hline & {$\left[\mathrm{M}-\mathrm{H}^{-}\right.$} & $I$ & II & III & $I V$ \\
\hline $1004(1013)$ & 1004 & & & & \\
\hline 924 & 924 & & & & \\
\hline \multicolumn{6}{|l|}{$766(775)$} \\
\hline $675(680)$ & 675 & $675(680)$ & & & \\
\hline $657(660)$ & 657 & $657(660)$ & $657(660)$ & & \\
\hline $595(599)$ & & 595 (595) & & $595(599)$ & \\
\hline \multirow[t]{2}{*}{$577(579)$} & 577 & $577(579)$ & $577(579)$ & & $577(579)$ \\
\hline & 488 & & & & \\
\hline $426(432)$ & 426 & & & & \\
\hline $408(412)$ & 408 & & & & \\
\hline \multirow[t]{2}{*}{$357(361)$} & & $357(361)$ & $357(361)$ & $357\{361\}$ & \\
\hline & 328 & & & & \\
\hline \multirow[t]{4}{*}{$339(341)$} & & $339(341)$ & $339(341)$ & $339(341)$ & $339(341)$ \\
\hline & & & & $323\{326\}$ & \\
\hline & & $305(306)$ & $305(306)$ & & 305 (306) \\
\hline & 273 & & & & \\
\hline \multirow[t]{5}{*}{$271(271)$} & & $271(271)$ & $271(271)$ & $271\{271\}$ & $271(271)$ \\
\hline & & $208(209)$ & $208(209)$ & & $208(209)$ \\
\hline & & & & & 193 \\
\hline & & & & & $163(163)$ \\
\hline & & $159(160)$ & $159(160)$ & & \\
\hline
\end{tabular}

${ }^{a}$ Mass-to-charge ratio values in parentheses are from $\left[{ }^{2} \mathrm{H}\right]_{10}-(\mathrm{N}, \mathrm{O})$-palmitoyl CoA

${ }^{b}$ Mass-to-charge ratio values of precursor ions selected for $\mathrm{CID}$ are underlined.

'Source-formed ions observed in FAB MS. 

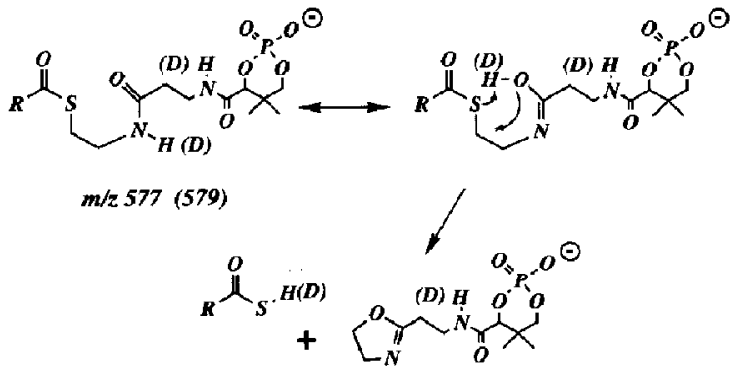

$\mathrm{m} / \mathrm{z}, 305(306)$

Scheme II

implies a deuterium transfer from the amide nitrogen, perhaps to form an oxazole ring (Scheme II). The fragment ion at $m / z 159$ is consistent with $\left[\mathrm{HP}_{2} \mathrm{O}_{6}\right]^{-}$ and this ion also shifted by $1 \mathbf{u}$ in the CID spectrum of the deuterated analog (Table 3). The other ion $(\mathrm{m} / \mathrm{z}$ 208 ) is a common product ion in the CID spectra of $I$, $I I$, and $I V$, and the formation of this ion most likely involves the loss of $\mathrm{RCOSCH}_{2} \mathrm{CH}_{2} \mathrm{NHCOCH}=\mathrm{CH}_{2}$ from $m / z 577$ (IV) perhaps via a McLafferty-like $\gamma$-proton rearrangement (Scheme III). The resultant structure of this ion retains only one exchangeable hydrogen and is in agreement with a $1 \mathrm{u}$ shift to $\mathrm{m} / \mathrm{z}$ 209 in the CID spectrum of the deuterated analog (Table 3).

lon II ( $m / 2$ 657). The CID spectrum of this ion from palmitoyl CoA was quite similar to that of $I$, except that the abundance of $I V, m / z 577$, was very low in this case and no type $I I I, m / z$ 595, was observed. All other product ions listed in Table 3 are as described above in the CID spectrum of $I, m / z 675$.

Ion III (m/z 595). A single abundant ion, $m / z 357$

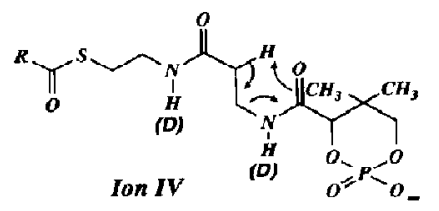

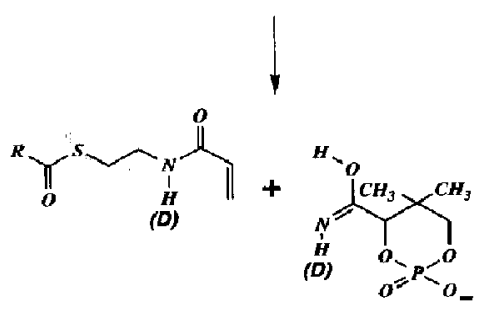

$m / z 208$ (209)
(D) dominated the product ion spectrum of III as shown in Figure 4. This is a common ion obseryed in CID of III from all other acyl CoAs and is formed by the neutral loss of alkylketene, in this case hexadecylketene ( $238 \mathrm{u}$ ). Low abundance ions observed in the spectrum included $m / z \quad 339,323$, and 271 . Of these, $m / z 339(E)$ and $271(V)$ have been discussed above. The ion at $m / z$ 32.3, which is observed in the CID of III from all other acyl CoAs, is derived by loss of the acylthioacid (RCOSH, $272 \mathrm{u}$ ) as in the formation of $m / z 305$ in the CID spectrum of $I$ (Scheme II). This ion shifted by $3 \mathbf{u}$ in the CID of the deuterated analog, which again implied the transfer of a deuterium atom from an amide to form the neutral thioacid.

Ion IV ( $m / z 577)$. The CID behavior of $I V$ was similar to that observed with III in that a single abundant product ion was formed by the loss of the neutral alkylketene, resulting in the ion at $m / z 339(E)$. Other ions observed in the spectrum (Table 3) were of very low abundance.

Ion B ( $m / z 426)$. This ion, which does not contain the acyl group, produced four ions with collisional activation. The ions observed were $m / z 408$ (loss of water), 328 (loss of $\left.\mathrm{H}_{2} \mathrm{PO}_{4}\right), 159\left(\left[\mathrm{HP}_{2} \mathrm{PO}_{6}\right]^{-}\right.$), and 134 ([adenine $-\mathrm{H}]^{-}$) (data not shown).

Ion $\mathrm{C}(\mathrm{m} / \mathrm{z}$ 408). The product ion spectrum of $\mathrm{C}$ contained only one ion not observed in decomposition of $B$. This ion, $m / z$ 273, which was also observed in the product ion spectrum of the $[\mathrm{M}-\mathrm{H}]^{-}$, is most likely due to the loss of adenine, $135 \mathrm{u}$ (data not shown).

Ion $V(m / z 271)$. The acyl thiolate anion, [RCOS] ${ }^{-}$, is unique for each acyl CoA studied and is analogous to the carboxylate anion derived from negative ion FAB/MS of fatty acids and phospholipids [14-16]. The $\mathrm{CID}$ of $V$ did not yield many product ions. However,

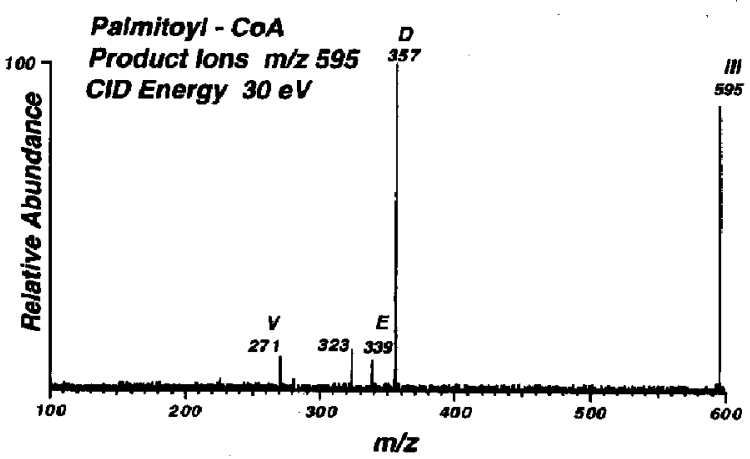

Figure 4. Product ions obtained following collision induced dissociation (30 eV collision energy, 0.5 m'Torr argon) of $\mathrm{m} / \mathrm{z}$ 595. Type III ions from palmitoyl CoA. 
increasing the collision offset $\left(Q_{2}\right)$ potential to $100 \mathrm{~V}$ did produce a low abundance series of product ions differing in $14 \mathrm{u}$, beginning at $m / z 60, \mathrm{SCO}^{-}$. The abundance of this ion series was not sufficient to determine double bond positions in unsaturated acyl CoAs, as has been demonstrated in the high energy CID of carboxylate anions from fatty acids [14-16]. Precursor ion spectra in the formation of this ion $(\mathrm{m} / z$ 271) contained only one abundant ion, $I V$, and to a much lesser extent, III $(\mathrm{m} / \mathrm{z} 577$ and 595, respectively, for palmitoyl CoA).

\section{Analysis of Acyl CoA Mixtures by FAB /MS / MS}

The product ion spectra of $I I I$ and $I V$ from all acyl $C a A$ species studied contained abundant ions from the loss of alkylketene at $m / z \quad 357$ and 339 ( $D$ and $E$, respectively). The precursor scans of these ions, $D$ and $E$, also contained only abundant ions corresponding to $I I I$ and $I V$, respectively (data not shown). As $D$ and $E$ are fragment ions common to all acyl $C O A$ species, precursor scans of these ions during FAB/CID of mixtures of acyl CoA thioesters contained ions diagnostic of each acyl $\mathrm{COA}$ species in the mixture.

Figure $5 \mathrm{a}$ shows the negative ion $\mathrm{FAB} / \mathrm{MS}$ of a mixture of seven standard acyl CoA thioesters with the inset expanding the mass-to-charge ratio region containing the $I I I$ and $I V$ type ions from each of the CoA species present. A much simpler spectrum results from CID and precursor scans of $m / z$ 339, $E$ (Figure 5b). In this spectrum, only $I V$ ions are detected and the expanded region in Figure $5 \mathrm{~b}$ reveals ions clearly indicating each acyl CoA species present in the mixture.

An alternate strategy would be to use CID and tandem mass spectrometric analysis to search a mixture of acyl CoA esters for the presence of one or more specific molecular species. Neutral loss scans of a specific alkylketene would detect only the transition of the $I I I$ and $I V$ type ions to $E(m / z 357)$ and $D(m / z 339)$ ions associated with that specific molecular species. This is demonstrated in Figure $5 c$ with the same acyl $\mathrm{CoA}$ mixture employing neutral loss scans of $238 \mathrm{u}$ (loss of hexadecylketene), a specific loss for palmitoyl CoA. As shown only $m / z 595$ and 577 (III and $I V$, respectively from palmitoyl $\mathrm{CoA}$ ) are observed (Figure 5c).

\section{Discussion}

The negative ion FAB/MS spectra of long-chain acyl $C O A$ thioesters are characterized by the formation of abundant $\left[\mathrm{M}-\mathrm{H}^{-}\right.$and two distinct classes of fragment ions, one class which retains the acyl group and another class which is related to CoA. For the identification of specific acyl $\mathrm{COA}$ species the first series of fragment ions $(I-V)$ are obviously more important as these ions retain the long-chain fatty acyl group. With
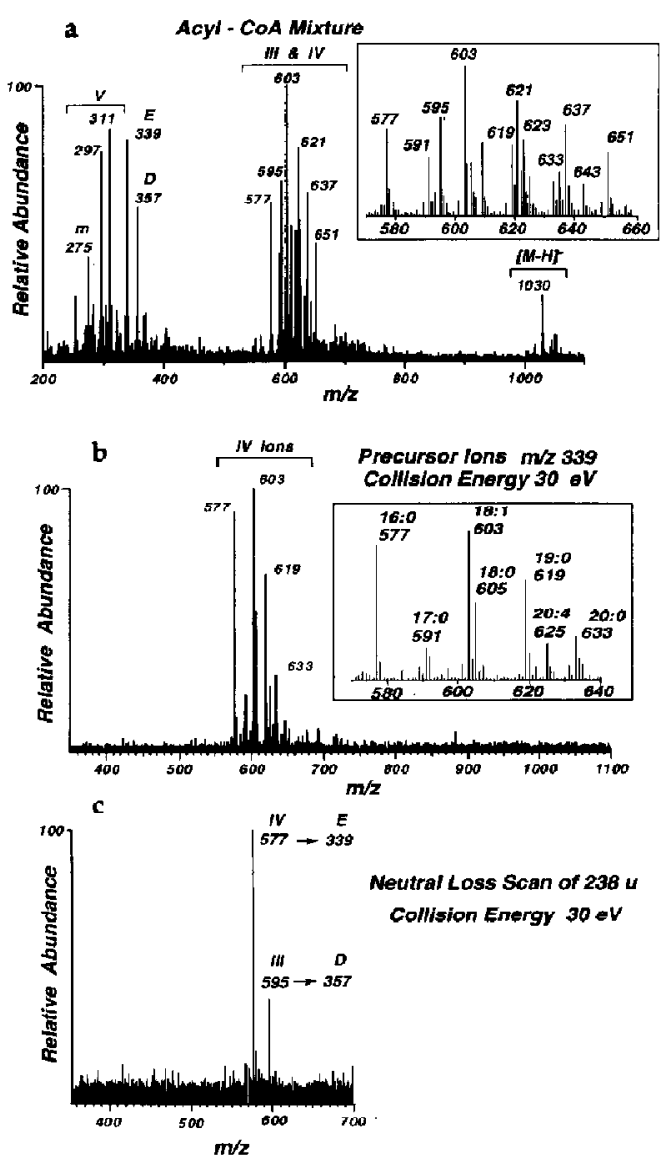

Figure 5. (a) FAB/MS of a mixture of acyl CoAs containing C16:0, C17:0, C18:1, C18:0, C19:0, C20:0, and C20:4 acyl species. The spectrum was obtained from a glycerol matrix and the letter m designates matrix ions; (b) precursor ions of $m / z 339$ obtained following collision induced dissociation $(30 \mathrm{eV}$ collision energy. $0.5 \mathrm{mTorr}$ argon) of source formed ions, $m / z$ 350-1100 from the same mixture of acyl CoA species; and (c) precursor ions of the neutral loss of hexadecylketene, $238 \mathrm{u}$, obtained following collision induced dissociation ( $30 \mathrm{eV}$ collision energy, $0.5 \mathrm{mTorr}$ argon) of source formed ions, $m / z 300-700$, from the same mixture of acyl CoA species.

negative ionization the class of fragment ions retaining the acyl group are much more abundant than the CoA related class of ions (Table 1 ).

The most probable site of the initial negative ionization is one of the three phosphate groups and many of the fragment ions are derived from fragmentation of a phospho anhydride or ester bond and subsequent losses of either water or alkylketene. As has been described above, the ions which retain the acyl group in the spectrum of palmitoyl $\mathrm{CoA}$ appear at $m / z 675$, 657,595 , and 577 . Ions formed from these by loss of alkylketene are $m / z 357$ and 339. Fragment ions which retain the adenine group are observed at $\mathrm{m} / \mathrm{z} 426$ and 408 (Figure 1). In contrast to these ions observed following FAB ionization, tandem mass spectrometry of 
the $\left[\mathrm{M}-\mathrm{H}^{-}\right.$, from palmitoyl $\mathrm{CoA}(m / z$ 1004), yielded the adenine-containing ions as major products and the acyl-containing ions were of low abundance or not detected. In addition, the most abundant fragment ion observed in the FAB/MS spectrum, the thiolate anion ( $m / z$ 271), was not observed at all (as well as the lesser abundant ion at $m / z 766$ ). These results suggested that the formation of many characteristic ions observed in direct FAB analysis occurred during the desorption process. Although the excitation and decomposition of source-formed ions can be quite different from collisional activation with respect to energy and time considerations, other observations argue that these are not the only parameters affecting fragmentation. The observed abundances of fragment ions in the $\mathrm{FAB} / \mathrm{MS}$ spectra were dependent upon the matrix employed. With relatively basic matrices such as diethanolamine and triethanolamine, the most abundant fragment ions formed were the thiolate anions $(V)$. In contrast, with less basic matrices such as glycerol and thioglycerol, the most abundant ions formed were the type $I I I$ and IV (Table 2) which involve fragmentation of the phospho anhydride bond. These observations argue for an active role of either matrix molecules or matrix-derived anions in the formation of fragment ions rather than ionization solely at a phosphate residue. Such interactions occurring in the selvedge region during desorption and ionization of both positive and negative ions have been reviewed [17] and previously observed in the negative ion FAB /MS of another phosphate-containing lipid, glycerophusphocholine [18].

Fragmentation of the central phospho anhydride bond can form either an acylpantetheinophosphate anion or a diphosphoadenosine anion. The abundant type III ions ( $m / z 595$ in palmitoyl CoA) observed in the FAB/MS spectrum suggest that the formation of the acylpantetheinophosphate anion was greatly favored over the formation of the diphosphoadenosine anion, $m / z$ 426. Fragmentation on either side of the anhydride bond may be equally favored, but the strongly basic amino sites in adenine may also abstract a proton from other matrix molecules to form a neutral zwitterionic diphosphoadenosine species. This would result in the observed greater abundance of the acyl-containing species. In the absence of matrix molecules, fragmentation of this anhydride bond also occurred, as in the CID spectrum of the $[\mathrm{M}-\mathrm{H}]^{-}$from palmitoyl $\mathrm{CoA}$ (Figure 2). In this decomposition mode charge retention by the diphosphoadenosine was favored. Without the presence of matrix molecules during CID, formation of the diphosphoadenosine anion may be favored with charge localized over two phosphate groups, while the acylpantetheinophosphate contains only one such group to stabilize the charge.

Positive ionization FAB/MS of acyl CoAs also yielded abundant $[\mathrm{M}+\mathrm{H}]^{+}$and acyl retaining fragment ions $[10,11]$. Two major differences were observed between positive and negative ion spectra: the base peak observed with positive ionization was protonated adenine, $m / z$ 136, while the base peak with negative ionization was typically the acyl thiolate anion, $V$. The acyl thiolate anion was not observed in the positive ion FAB spectrum of acyl CoAs, and the formation of this ion in the negative ionization mode, especially when enhanced by the presence of a basic matrix, may be particularly useful in the identification of isomeric long-chain acyl CoAs. Clearly, tandem mass spectrometric analysis of both positive and negative ions generated from long-chain fatty acyl CoA esters provide structurally useful and complementary information.

The use of CID and tandem mass spectrometric techniques with negative ion formation will undoubtedly be useful in the analysis of complex mixtures of acyl CoAs. The unique relationship between type $I V$ and $E$ or $I I I$ and $D$ ions which involve the transition from acyl-containing ions to only CoA-containing ions allows the development of tandem mass spectrometry protocols similar to those developed for the analysis of phospholipid mixtures. Such techniques have been used with good success with phosphocholine mixtures to determine both molecular species $[19,20]$ and to identify novel individual species [21]. These techniques are directly adaptable to mixtures of acyl CoAs, using either precursor or neutral loss scans.

\section{Acknowledgments}

This work was supported, in part, by grants from the National Institutes of Health (HL34303) and Amgen Inc.

\section{References}

1. Black, K.; Vance, D. Ann. Rev. Biochem. 1977, 46, 263-298.

2. Waku, K. Biochim. Biophys. Acta 1992, 1124, 101-111.

3. Vance, D. In Biochemistry of Lipids and Membranes, Vance, D. E., and Vance, J. E., Eds. Benjamin Cummings: Menlo Park, CA 1985; Chapter 8, pp. 242-270.

4. Venable, M. E.; Zimmerman, G. A.; McIntyre, T. M.; Prescott, S. M. I. Lipid Res. 1993, 34, 691-702.

5. Shirley, M. A.; Murphy, R. C. J. Biol. Chent. 1990, 265, 16288-16295.

6. Yamaoka, A.; Sumimoto, H.; Isobe, R.; Minakami, S. Biochem. Biophys. Res. Commun. 1988, 154, 1248-1252.

7. Kolis, S. T.; Rostma, E. J.; Williams, T. H.; Sasso, G. J. Drug Metab. Disp. 1986, 14, 465-470.

8. Gregus, F.; Fekete, T.; Varga, F.; Klaasen, C. D. Drug Metab. Disp. 1992, 20, 234-240.

9. Wolf, B. A.; Conrad-Kessel, W.; Turk, J. J. Chromatogr. 1990, $509,325-332$.

10. 'Iaylor, D. C.; Weber, N.; Hogge, L. R.; Underhill, E. W. Anal. Biochem. 1990, 184, 311-316.

11. Norwood, D. L,; Bus, C. A.; Millington, D. S. J. Chromatogr. 1990, 527, 289-301.

12. Millington, D. S.; Norwood, D. L.; Kodo, N.; Moore, R.; Green, M. D.; Berman, J. J. Chromatogr. 1991, 562, 47-58.

13. Li, J.; Norwood, D. L.; Mao, L.-F.; Schultz, H. Biochem. 1991, $30,388-394$. 
14. Jensen, N. J.; Tomer, K. B.; Gross, M. L. J. Am. Chem. Soc. 1985, 107, 1863-1868.

15. Adams, J.; Gross, M. L. Anal. Chem. 1987, 59, 1576-1582.

16. Jensen, N. J.; Tomer, K. B.; Gross, M. L. Lipids 1986, 21, $580-588$.

17. Detter, L. D.; Hand, O. W.; Cooks, R. G.; Walton, R. A. Mass Spectrom. Rev, 1988, 7, 465-502.
18. Zirrolli, J. A.; Clay, K. C.; Murphy, R. C. Lipids 1991, 26, 112-116.

19. Kayganich, K. A.; Murphy, R. C. I. Am. Soc. Mass Spectrom. 1991, 2, 45-54.

20. Cole, M. J.; Enke, C. G. Anal. Chem. 1991, 63, 1032-1038.

21. Kayganich-Harrison, K. A.; Rose, D. A.; Murphy, R. C.; Morrow, J. D.; Roberts, L. J. II, J. Lipid Research 1993, 34, $1229-1235$. 